\title{
Expansion Theorem for the Linearized Fokker-Planck Equation
}

\author{
JORDAN D. LEWIS* \\ Department of Nuclear Engineering, University of Michigan, Ann Arbor, Michigan
}

(Received 22 December 1965)

\begin{abstract}
The linearized Fokker-Planck kinetic equation for each component of a homogeneous, nondegenerate, fully ionized plasma is separated by means of a spherical harmonic expansion into an infinite set of singular integro-differential equations. Each equation is shown to generate a continuous set of eigenfunctions, for which asymptotic high-speed forms are found. By extending the theory of singular differential equations an expansion formula is developed, which is shown to be complete with respect to functions square integrable in velocity space.
\end{abstract}

\section{INTRODUCTION}

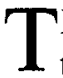
HE Fokker-Planck kinetic equation may be used to describe the temporal behavior of a homogeneous, nondegenerate, fully ionized plasma. $^{1-3}$ The Fokker-Planck equation has been derived by several authors (see e.g., the discussion in Robinson and Bernstein $^{3}$ ), starting with various levels of sophistication in kinetic theory. Whatever the starting point, the results are essentially the same. ${ }^{2.3}$ For our purposes, it is convenient to write the Fokker-Planck equation in the form ${ }^{2,3}$

$\frac{\partial F_{a}}{\partial t}=\sum_{b} \frac{\partial}{\partial \mathbf{v}} \cdot \int d^{3} v^{\prime}\left(F_{b}^{\prime} \frac{\partial F_{a}}{\partial \mathbf{v}}-\frac{m_{a}}{m_{b}} F_{a} \frac{\partial F_{b}^{\prime}}{\partial \mathbf{v}^{\prime}}\right) \cdot \mathbf{Q}_{a b}\left(\mathbf{v}, \mathbf{v}^{\prime}\right)$.

Here $F_{a}(\mathbf{v}, t) d^{3} v$ is the expected number of particles of kind $a$ in the velocity space volume element $d^{3} v$ about $\mathrm{v}$ at time $t$,

$$
\mathbf{Q}_{a b}=\Gamma_{a b} g^{-3}(\mathbf{I}-\mathbf{g g}),
$$

where $\Gamma_{a b}$ is a positive constant, $\mathbf{g}=\mathbf{v}-\mathbf{v}^{\prime}$, and $\mathbf{I}$ is the unit dyadic. We denote by $F_{j}^{\prime}$ the function $F_{j}\left(\mathbf{v}^{\prime}, t\right)$.

It is not difficult to show ${ }^{2}$ that $\partial F_{a} / \partial t=0$ if and only if $F_{a}$ and $F_{b}$ are the equilibrium, or Maxwell, distributions $F_{a 0}, F_{b 0}$. In the vicinity of equilibrium we may write $F_{j}=F_{j 0}\left[1+f_{j}(\mathbf{v}, t)\right]$. Neglecting terms quadratic in $f_{j}$ we then obtain from (1) the linear equation

$$
F_{a 0} \frac{\partial f_{a}}{\partial t}=\sum_{b} \frac{\partial}{\partial \mathbf{v}} \cdot \int d^{3} v^{\prime} F_{b 0}^{\prime} F_{a 0}\left(\frac{\partial f_{a}}{\partial \mathbf{v}}-\frac{m_{a}}{m_{b}} \frac{\partial f_{b}^{\prime}}{\partial \mathbf{v}^{\prime}}\right) \cdot \mathbf{Q}_{a b}
$$

* Present address: The Battelle Development Corporation, Columbus, Ohio.

I M. Rosenbluth, W. M. MacDonald and D. L. Judd, Phys. Rev. 107, 1 (1957).

2 D. C. Montgomery and D. A. Tidman, Plasma Kinetic Theory (McGraw-Hill Book Company, Inc., New York, 1964), Chaps. 2 and 3.

${ }^{3}$ B. B. Robinson and I. B. Bernstein, Ann. Phys. (N.Y.) 18, 110 (1962).
We refer to the quantity $F_{j 0} f_{j}$ as the perturbation from equilibrium.

Equation (2) as it stands is in fact a pair of coupled equations for $f_{a}$ and $f_{b}$. Due to the quite small value of the electron-ion mass ratio, the equations however are only very weakly coupled. Thus, for example, the effect of the ion perturbation on the electron perturbation is small when compared with the effect of the ions and electrons in the unperturbed equilibrium distributions.

In the following we consider the equation for the ions. The treatment of the electron equation is quite similar, and the modifications necessary for this case are indicated later (Sec. V). In the approximation $m_{i} \gg m_{\epsilon}$ it can be shown ${ }^{3}$ that the ions act like a single component gas. In this case Eq. (2) becomes, for the ions,

$$
F_{i 0} \frac{\partial f_{i}}{\partial t}=\frac{\partial}{\partial \mathbf{v}} \cdot \int d^{3} v^{\prime} F_{i 0}^{\prime} F_{i 0}\left(\frac{\partial f_{i}}{\partial \mathbf{v}}-\frac{\partial f_{i}^{\prime}}{\partial \mathbf{v}^{\prime}}\right) \cdot \mathbf{Q}_{i i} .
$$

Our purpose here is to develop an expansion theorem based on (3). We follow the standard method of assuming solutions to (3) of the form $f(\mathbf{v}, t)=$ $g_{\lambda}(\mathbf{v}) \exp (-\lambda t)$. This reduces (3) to the form $L(\mathbf{v}) g_{\lambda}(\mathbf{v})=-\lambda g_{\lambda}(\mathbf{v})$, where $L$ is a three-dimensional integro-differential operator. In Sec. I we show that (3) requires $\operatorname{Re} \lambda>0$ and $\operatorname{Im} \lambda=0$ as we would expect physically. In Sec. II, we introduce a spherical harmonic expansion which replaces the three-dimensional equation by an infinite set of uncoupled equations, $L_{l m} g_{l m}=-\lambda_{l m} g_{l m}$, where $L_{l m}(v)$ is a singular integro-differential operator. These are cast into a self-adjoint form in Sec. III by introducing a suitable algebraic transformation on the functions $g_{l m}\left(v, \lambda_{l m}\right)$. With boundary conditions obtained by combining ( 3 ) with the conservation laws, we proceed to find the eigenvalue spectrum which is continuous and for $l=0,1$ consists of all $\lambda \geq 0$ and for $l \geq 2$ consists of all $\lambda>0$. 
The expansion theorem is developed in Sec. IV. (Although the spectral resolution theorem implies the existence of an expansion theorem for self-adjoint operators, there remains the nontrivial task of constructing the expansion explicitly.) Since $L_{l m}$ is singular at $v=0$ and $v \rightarrow \infty$, we temporarily replace the interval $0 \leq v<\infty$ by the interval $0<v_{1} \leq$ $v \leq v_{2}<\infty$ and show that $L_{l m}$ generates a complete orthonormal set on this interval. To return to the original interval and thus obtain the desired expansion theorem, we use the above completeness property together with an extension of the theory of singular differential equations. This finally yields a set of functions $\left\{\Psi_{l m}\left(\mathbf{v}, \lambda_{l m}\right)\right\}$ that is complete with respect to functions square integrable in velocity space. Since the spectrum is continuous, the expansion has the form of a generalized Fourier integral. For $v \gg$ $\left(3 k T / m_{i}\right)^{\frac{1}{2}}$ we have found asymptotic forms of the expansion functions.

\section{PROPERTIES OF THE KINETIC EQUATION}

For convenience we drop the subscript " $i$ " from $F_{0 i}$ and $f_{i}$. If $f$ satisfies the conditions

$$
\lim _{v \rightarrow 0} v f=0, \quad \lim _{v \rightarrow \infty} F_{0} \frac{\partial f}{\partial v}=0,
$$

it is possible to show that the linear kinetic equation (3) conserves number, momentum, and kinetic energy densities.

Introducing $f(\mathbf{v}, t)=g_{\lambda}(\mathbf{v}) \exp (-\lambda t)$ in (3), we find

$$
-\lambda F_{0} g_{\lambda}=\frac{\partial}{\partial \mathbf{v}} \cdot \int d^{3} v^{\prime} F_{0} F_{0}^{\prime}\left(\frac{\partial g_{\lambda}}{\partial \mathbf{v}}-\frac{\partial g_{\lambda}^{\prime}}{\partial \mathbf{v}^{\prime}}\right) \cdot \mathbf{Q} .
$$

Multiplying (5) by $g_{\lambda}^{*}$, where ${ }^{*}$ denotes complex conjugate, and then integrating over $\mathbf{v}$ we have, after integrating by parts,

$$
\begin{gathered}
\lambda \int d^{3} v F_{0}\left|g_{\lambda}\right|^{2}=\int d^{3} v \frac{\partial g_{\lambda}^{*}}{\partial \mathbf{v}} \cdot \int d^{3} v^{\prime} F_{0} F_{0}^{\prime}\left(\frac{\partial g_{\lambda}}{\partial \mathbf{v}}-\frac{\partial g_{\lambda}^{\prime}}{\partial \mathbf{v}^{\prime}}\right) \cdot \mathbf{Q} \\
-\int d^{3} v \frac{\partial}{\partial \mathbf{v}} \cdot g_{\lambda}^{*} \int d^{3} v^{\prime} F_{0} F_{0}^{\prime}\left(\frac{\partial g_{\lambda}}{\partial \mathbf{v}}-\frac{\partial g_{\lambda}^{\prime}}{\partial \mathbf{v}^{\prime}}\right) \cdot \mathbf{Q}
\end{gathered}
$$

The second term on the right in (6) vanishes provided $g_{\lambda}$ satisfies the second of conditions (4) and

$$
\lim _{v \rightarrow 0} v^{\frac{1}{2}} g_{\lambda}(v)=0
$$

Assuming these conditions hold, we exchange $v$ and $v^{\prime}$ in (6), noting that $\mathbf{Q}\left(\mathbf{v}, \mathbf{v}^{\prime}\right)=\mathbf{Q}\left(\mathbf{v}^{\prime}, \mathbf{v}\right)$. We add the result to (6), obtaining

$$
\begin{aligned}
& 2 \lambda \int d^{3} v F_{0}\left|g_{\lambda}\right|^{2} \\
& \quad=\iint d^{3} v d^{3} v^{\prime} F_{0} F_{0}^{\prime}\left(\frac{\partial g_{\lambda}^{*}}{\partial \mathbf{v}}-\frac{\partial g_{\lambda}^{\prime *}}{\partial \mathbf{v}^{\prime}}\right) \cdot \mathbf{Q} \cdot\left(\frac{\partial g_{\lambda}}{\partial \mathbf{v}}-\frac{\partial g_{\lambda}^{\prime}}{\partial \mathbf{v}^{\prime}}\right) .
\end{aligned}
$$

Since $\mathbf{Q}$ is a positive quadratic form, it follows that the right side of $(8)$ is positive or zero. Hence $\operatorname{Re} \lambda \geq 0$, and clearly $\operatorname{Im} \lambda=0$.

Employing standard methods ${ }^{4}$ we can find from (8) the most general form of $g_{\lambda}$ when $\lambda=0$;

$$
g_{0}(\mathbf{v})=a_{1} v^{2}+\mathbf{a}_{2} \cdot \mathbf{v}+a_{3},
$$

with $a_{1}, \mathbf{a}_{2}$, and $a_{3}$ arbitrary constants.

\section{EXPANSION IN SPHERICAL HARMONICS}

From (5) we have

$$
\begin{aligned}
-\lambda F_{0} g_{\lambda}=\frac{\partial}{\partial \mathbf{v}} \cdot\left(F_{0} \frac{\partial g_{\lambda}}{\partial \mathbf{v}} \cdot \int d^{3} v^{\prime} F_{0}^{\prime} \mathbf{Q}\right) & \\
& -\frac{\partial}{\partial \mathbf{v}} \cdot\left(F_{0} \int d^{3} v^{\prime} F_{0}^{\prime} \frac{\partial g_{\lambda}^{\prime}}{\partial \mathbf{v}^{\prime}} \cdot \mathbf{Q}\right)
\end{aligned}
$$

To perform a parts integration on the second term, consider the quantity

$$
\begin{aligned}
\frac{\partial}{\partial \mathbf{v}^{\prime}} \cdot\left(F_{0}^{\prime} g_{\lambda}^{\prime} \mathbf{Q}\right)=F_{0}^{\prime} g_{\lambda}^{\prime} & \frac{\partial}{\partial \mathbf{v}^{\prime}} \cdot \mathbf{Q} \\
& +F_{0}^{\prime} \frac{\partial g_{\lambda}^{\prime}}{\partial \mathbf{v}^{\prime}} \cdot \mathbf{Q}+g_{\lambda}^{\prime} \frac{\partial F_{0}^{\prime}}{\partial \mathbf{v}^{\prime}} \cdot \mathbf{Q} .
\end{aligned}
$$

With $F_{0}=N_{0}\left(\alpha_{i} / \pi\right)^{\frac{3}{2}} \exp \left(-\alpha_{i} v^{2}\right), \quad \alpha_{i}=m_{i} / 2 k T$, we have $\partial F_{0}^{\prime} / \partial \mathbf{v}^{\prime}=-2 \alpha_{i} \mathbf{v}^{\prime} F_{0}^{\prime}$. Also $\mathbf{v}^{\prime} \cdot \mathbf{Q}=\mathbf{v} \cdot \mathbf{Q}$, so (10) gives

$$
\begin{aligned}
& \int d^{3} v^{\prime} F_{0}^{\prime} \frac{\partial g_{\lambda}^{\prime}}{\partial \mathbf{v}^{\prime}} \cdot \mathbf{Q}=\int d^{3} v^{\prime} \frac{\partial}{\partial \mathbf{v}^{\prime}} \cdot\left(F_{0}^{\prime} g_{\lambda}^{\prime} \mathbf{Q}\right) \\
& -\int d^{3} v^{\prime} F_{0}^{\prime} g_{\lambda}^{\prime} \frac{\partial}{\partial \mathbf{v}^{\prime}} \cdot \mathbf{Q}+2 \frac{\partial}{\partial \mathbf{v}} \cdot \int d^{3} v^{\prime} F_{0}^{\prime} g_{\lambda}^{\prime} \mathbf{Q} .
\end{aligned}
$$

The first term on the right in (11) vanishes if $g_{\lambda}$ satisfies (4). Using the relations

$$
\mathbf{Q}=\Gamma_{i i} \frac{\partial^{2} g}{\partial \mathbf{v} \partial \mathbf{v}}, \frac{\partial}{\partial \mathbf{v}^{\prime}} \cdot \mathbf{Q}=-2 \Gamma_{i i} \frac{\partial}{\partial \mathbf{v}}\left(\frac{1}{g}\right),
$$

and introducing a dimensionless time $\tau$ and dimensionless velocity $\mathrm{c}$ in (3),

$$
\tau\left(=\tau_{i}\right)=4 \pi N_{0} \Gamma_{i i}\left(\alpha_{i} / \pi\right)^{\frac{3}{2}} t, \quad \mathbf{c}=\alpha_{i}^{\frac{1}{2}} \mathbf{v},
$$

the kinetic equation takes the form

$$
\begin{aligned}
- & 4 \pi \lambda e^{-c^{2}} g_{\lambda}(\mathbf{c}) \\
= & \frac{\partial}{\partial \mathbf{c}} \cdot\left(e^{-c^{2}} \frac{\partial g_{\lambda}}{\partial \mathbf{c}} \cdot \frac{\partial^{2}}{\partial \mathbf{c} \partial \mathbf{c}} \int d^{3} c^{\prime} e^{-c^{\prime 2}}\left|\mathbf{c}-\mathbf{c}^{\prime}\right|\right) \\
& -2 \frac{\partial}{\partial \mathbf{c}} \cdot\left(e^{-c^{2}} \frac{\partial}{\partial \mathbf{c}} \int d^{3} c^{\prime} e^{-c^{\prime 2}} g_{\lambda}^{\prime}\left|\mathbf{c}-\mathbf{c}^{\prime}\right|^{-1}\right) \\
& -2 \frac{\partial}{\partial \mathbf{c}} \cdot\left(\mathbf{c} e^{-c^{2}} \cdot \frac{\partial^{2}}{\partial \mathbf{c} \partial \mathbf{c}} \int d^{3} c^{\prime} e^{-c^{\prime 2}} g_{\lambda}^{\prime}\left|\mathbf{c}-\mathbf{c}^{\prime}\right|\right) .
\end{aligned}
$$

\&eference 2, p. 85. 
The time $\tau$ is measured in units of the "Spitzer selfcollision time," ${ }^{5}$ and $c=|c|$ is in units of the rms thermal speed.

Equation (12), in three dimensions, may be replaced by a set of uncoupled equations in one dimension by introducing the spherical harmonic expansion

$$
g_{\lambda}(\mathbf{c})=\sum_{l=0}^{\infty} \sum_{m=-l}^{l} g_{l m}\left(c, \lambda_{l m}\right) Y_{l}^{m}(\theta, \phi) .
$$

We find (see Appendix A)

$$
\begin{gathered}
\int d^{3} c^{\prime} e^{-c^{\prime 2}} g_{\lambda}^{\prime}\left|\mathbf{c}-\mathbf{c}^{\prime}\right|^{-1}=\sum_{l, m} \frac{4 \pi}{2 l+1} Y_{l}^{m} R_{l m}, \\
\int d^{3} c^{\prime} e^{-c^{\prime 2}} g_{\lambda}^{\prime}\left|\mathbf{c}-\mathbf{c}^{\prime}\right|=\sum_{l, m} \frac{4 \pi}{4 l^{2}-1} Y_{l}^{m} S_{l m}, \\
\int d^{3} c^{\prime} e^{-c^{\prime 2}}\left|\mathbf{c}-\mathbf{c}^{\prime}\right|=4 \pi T,
\end{gathered}
$$

where

$$
\begin{aligned}
& R_{l m}(c)= \int_{0}^{c} d c^{\prime} c^{\prime}\left(\frac{c^{\prime}}{c}\right)^{l+1} e^{-c^{\prime 2}} g_{l m}^{\prime}+\int_{c}^{\infty} d c^{\prime} c^{\prime}\left(\frac{c}{c^{\prime}}\right)^{l} e^{-c^{\prime 2}} g_{l m}^{\prime}, \\
& S_{l m}(c)= \int_{0}^{c} d c^{\prime} c^{\prime 3}\left(\frac{c^{\prime}}{c}\right)^{l-1}\left[\frac{2 l-1}{2 l+3}\left(\frac{c^{\prime}}{c}\right)^{2}-1\right] e^{-0^{\prime 2}} g_{l m}^{\prime} \\
&+\int_{c}^{\infty} d c^{\prime} c^{\prime 3}\left(\frac{c}{c^{\prime}}\right)^{l}\left[\frac{2 l-1}{2 l+3}\left(\frac{c}{c^{\prime}}\right)^{2}-1\right] e^{-c^{\prime 2}} g_{l m}^{\prime}, \\
& T(c)=\frac{1}{4} e^{-c^{2}}+\left(c+\frac{1}{2 c}\right) \frac{\pi^{\frac{1}{2}}}{4} \operatorname{erf}(c),
\end{aligned}
$$

with

$$
\operatorname{erf}(c) \equiv 2 \pi^{-\frac{1}{2}} \int_{0}^{c} d x \exp \left(-x^{2}\right)
$$

The expressions for $R_{l m}$ and $S_{l m}$ were found by Rosenbluth et al.,1 using a different method, for the axially symmetric case $m=0$.

Combining (12)-(15) we find

$$
\begin{aligned}
-\lambda e^{-c^{2}} & \sum_{l, m} Y_{l}^{m} g_{l m} \\
= & \sum_{l, m} \frac{\partial}{\partial \mathbf{c}} \cdot\left(e^{-c^{2}} \frac{\partial}{\partial \mathbf{c}}\left(Y_{l}^{m} g_{l m}\right) \cdot \frac{\partial^{2} T}{\partial \mathbf{c} \partial \mathbf{c}}\right) \\
& -\sum_{l, m} \frac{2}{2 l+1} \frac{\partial}{\partial \mathbf{c}} \cdot\left(e^{-c^{2}} \frac{\partial}{\partial \mathbf{c}}\left(Y_{l}^{m} R_{l m}\right)\right) \\
& -\sum_{l, m} \frac{2}{4 l^{2}-1} \frac{\partial}{\partial \mathbf{c}} \cdot\left(\mathbf{c} e^{-c^{2}} \cdot \frac{\partial^{2}}{\partial \mathbf{c} \partial \mathbf{c}}\left(Y_{l}^{m} S_{l m}\right)\right) .
\end{aligned}
$$

Since $T(c)$ is isotropic we can write

$$
\partial^{2} T / \partial \mathbf{c} \partial \mathbf{c}=\hat{\mathbf{e}}_{c} \hat{\mathbf{e}}_{c} T^{\prime \prime}+\left(\mathbf{I}-\hat{\mathbf{e}}_{c} \hat{\mathbf{e}}_{c}\right) c^{-1} T^{\prime},
$$

where primes now denote total differentiation with respect to $c$. Performing the indicated angular differentiations in (16) and then employing the orthogonality

\footnotetext{
${ }^{5}$ L. Spitzer, Jr., Physics of Fully Ionized Gases (Interscience
} Publishers, Inc., New York, 1962), 2nd ed., pp. 132-136. property of the spherical harmonics, we obtain the "radial" equation

$$
\begin{gathered}
-\lambda_{l m} g_{l m}=T^{\prime \prime} \frac{d^{2} g_{l m}}{d c^{2}}+\left[T^{\prime \prime \prime}+\left(\frac{2}{c}-2 c\right) T^{\prime \prime}\right] \\
\times \frac{d g_{l m}}{d c}-\frac{l(l+1)}{c^{3}} T^{\prime} g_{l m} \\
-\frac{2}{2 l+1}\left[R_{l m}^{\prime \prime}+\left(\frac{2}{c}-2 c\right) R_{l m}^{\prime}-\frac{l(l+1)}{c^{2}} R_{l m}\right] \\
-\frac{2}{4 l^{2}-1}\left(c S_{l m}^{\prime \prime \prime}+\left(3-2 c^{2}\right) S_{l m}^{\prime \prime}\right. \\
\left.-\frac{l(l+1)}{c^{2}}\left(c S_{l m}^{\prime}-S_{l m}\right)\right)
\end{gathered}
$$

The index $m$ is clearly superfluous and is deleted in the following. Performing the primed differentiations in (17) we find

$$
\begin{aligned}
-\lambda_{l} g_{l}= & \left(\frac{\pi^{\frac{1}{2}}}{4 c^{3}} \operatorname{erf}(c)-\frac{1}{2 c^{2}} e^{-c^{2}}\right) \frac{d^{2} g_{l}}{d c^{2}} \\
& +\left[\left(\frac{1}{2 c^{3}}+\frac{2}{c}\right) e^{-c^{2}}-\left(\frac{1}{c^{4}}+\frac{2}{c^{2}}\right) \frac{\pi^{\frac{1}{2}}}{4} \operatorname{erf}(c)\right] \frac{d g_{l}}{d c} \\
& +\left[2 e^{-c^{2}}-\frac{l(l+1)}{c^{3}}\right. \\
& \left.\times\left(\frac{1}{4 c} e^{-c^{2}}+\left(1-\frac{1}{2 c^{2}}\right) \frac{\pi^{\frac{1}{2}}}{4} \operatorname{erf}(c)\right\rangle\right] g_{l} \\
& +\frac{4 c^{3}(l+1)(l+2)}{(2 l+1)(2 l+3)} \int_{0}^{c} d c^{\prime} e^{-c^{\prime 2}}\left(\frac{c^{\prime}}{c}\right)^{l+4} g_{l}\left(c^{\prime}\right) \\
& -\frac{4 c}{2 l+1}\left(1+\frac{c^{2} l(l-1)}{2 l-1}\right) \\
& \times \int_{0}^{c} d c^{\prime} e^{-c^{\prime 2}}\left(\frac{c^{\prime}}{c}\right)^{l+2} g_{l}\left(c^{\prime}\right) \\
& -\frac{4 c^{3} l(l-1)}{4 l^{2}-1} \int_{c}^{\infty} d c^{\prime} e^{-c^{\prime 2}}\left(\frac{c}{c^{\prime}}\right)^{l-3} g_{l}\left(c^{\prime}\right) \\
& -\frac{4 c}{2 l+1}\left(1-\frac{c^{2}(l+1)(l+2)}{2 l+3}\right) \\
& \times \int_{c}^{\infty} d c^{\prime} e^{-c^{\prime 2}}\left(\frac{c}{c^{\prime}}\right)^{l-1} g_{l}\left(c^{\prime}\right) .
\end{aligned}
$$

For boundary conditions we use conditions (4), which were obtained from the conservation laws. Although (7) is stronger than the first of conditions (4), we see below that the solutions of (18) which satisfy (4) also satisfy (7).

\section{THE SPECTRUM}

If we introduce the transformation

$$
g_{l}\left(c, \lambda_{l}\right)=c^{-1} e^{c^{2} / 2} \psi_{l}\left(c, \lambda_{l}\right),
$$


we obtain from (18) the formally self-adjoint equation $\frac{d}{d c}\left(P \frac{d \psi_{l}}{d c}\right)+\left(Q_{l}+\lambda_{l}\right) \psi_{l}+\int_{0}^{\infty} d c^{\prime} K_{l}\left(c, c^{\prime}\right) \psi_{l}\left(c^{\prime}\right)=0$,

with

$$
\begin{aligned}
P(c)= & \frac{\pi^{\frac{1}{2}}}{4 c^{3}} \operatorname{erf}(c)-\frac{1}{2 c^{2}} e^{-c^{2}}, \\
Q_{l}(c)= & \left(\frac{3}{c^{5}}-\frac{1}{c}\right) \frac{\pi^{\frac{1}{2}}}{4} \operatorname{erf}(c)+\left(\frac{7}{2}-\frac{3}{2 c^{4}}-\frac{1}{c^{2}}\right) e^{-c^{2}} \\
& -\frac{l(l+1)}{c^{3}}\left[\frac{1}{4 c} e^{-c^{2}}+\left(1-\frac{1}{2 c^{2}}\right) \frac{\pi^{\frac{1}{2}}}{4} \operatorname{erf}(c)\right], \\
K_{l}\left(c, c^{\prime}\right)= & \frac{4}{2 l+1} e^{-\frac{1}{2}\left(c^{2}+c^{\prime 2}\right)}\left[\frac{(l+1)(l+2)}{2 l+3}\left(\begin{array}{l}
c^{3} \\
c^{3}
\end{array}\right)\right. \\
& \left.-\left(\begin{array}{l}
c \\
c^{\prime}
\end{array}\right)-\frac{l(l+1)}{2 l-1}\left(\begin{array}{l}
c c^{\prime 2} \\
c^{\prime} c^{2}
\end{array}\right)\right]\left[\begin{array}{l}
\left(c / c^{\prime}\right)^{l} \\
\left(c^{\prime} / c\right)^{l}
\end{array}\right] \begin{array}{l}
\left(c \leq c^{\prime}\right) \\
\left(c^{\prime} \leq c\right)^{\prime}
\end{array}
\end{aligned}
$$

Transforming (4) via (19), we have

$$
\begin{aligned}
\lim _{c \rightarrow \infty} e^{-c^{2} / 2} \frac{d \psi_{l}}{d c} & =0, \\
\lim _{c \rightarrow 0} \psi_{l} & =0 .
\end{aligned}
$$

We later show (Appendix B) that the problem (20)(22) is self-adjoint.

The spectrum of (20) is that set of numbers $\left\{\lambda_{l}\right\}$ such that $(20)$ has nontrivial solutions which satisfy (21) and (22). In view of the self-adjoint property the $\lambda_{l}$ are real. We have already seen that $\lambda_{l}$ must be positive for $l \geq 2$, and positive or zero for $l=0,1$.

We can find the spectrum of (20) by first considering the related problem

$$
(d / d c)\left[P\left(d y_{l} / d c\right)\right]+\left(Q_{l}+\lambda_{l}\right) y_{l}=0
$$

with conditions on the functions $y_{l}(c)$ identical to (21) and (22). Clearly $P, d P / d c$, and $Q_{l}$ are bounded and continuous for all finite $c$ except possibly near $c=0$. For $c \ll 1$ we have

$$
\begin{gathered}
P(c)=\frac{1}{3}-\frac{1}{5} c^{2}+O\left(c^{4}\right) \\
Q_{l}(c)=\frac{17}{5}+O\left(c^{2}\right)-l(l+1)\left(\frac{1}{3 c^{2}}-\frac{1}{15}+O\left(c^{2}\right)\right) .
\end{gathered}
$$

In general we can write

$$
P(c)=\frac{1}{2 c^{3}} \int_{0}^{c} d x\left(e^{-x^{2}}-e^{-c^{2}}\right)
$$

and thus $P(c)>0$ for all $c<\infty$. It follows that for $l \neq 0,(23)$ has a regular singular point at $c=0$.

For $c$ small, (23) has the asymptotic solutions

$$
y_{l}(c) \simeq c^{l+1}, c^{-l} \quad(c \ll 1) .
$$

The first of these satisfies (22) for all $l$. It also satisfies the stronger condition obtained from (7),

$$
\lim (c \rightarrow 0) c^{-\frac{1}{2}} y_{l}(c)=0 .
$$

The second solution satisfies neither condition. This is clear for $l \neq 0$. For $l=0$, the second solution is a constant which cannot be zero since the solutions (24) are linearly independent.

For $c$ sufficiently large and for $\lambda_{l} \neq 0$, (23) takes the asymptotic form

$$
\frac{d^{2} y_{l}}{d c^{2}}-\frac{3}{c} \frac{d y_{l}}{d c}+\frac{4 \lambda_{l} c^{3}}{\pi^{\frac{1}{2}}} y_{l}=0 \quad\left(c \text { large, } \lambda_{l} \neq 0\right) .
$$

We find $\left(\lambda_{l}>0\right)$

$$
y_{l}(c) \simeq A_{l} c^{\frac{3}{4}} \cos \left(v_{l} c^{c^{2}}-\gamma_{l}\right),
$$

with $\nu_{l} \equiv 4 \lambda_{l}^{\frac{1}{2}} / 5 \pi^{\frac{1}{4}}$.

Given $l$ and $\lambda_{l}$, Eq. (23) has only one solution which satisfies the condition at $c=0$. This solution thus contains only one arbitrary constant and it follows that $A_{l}$ and $\gamma_{l}$ in (25) are not independent. Whatever the relation between $A_{l}$ and $\lambda_{l}$ is, (25) satisfies (21) for all positive $\gamma_{l}$. Hence the spectrum of (20) contains all positive $\lambda_{l}$.

To determine the spectrum of the problem in Eqs. (20)-(22), we first note that the solutions of the problem in Eqs. (21)-(23) do not exist in Hilbert space; hence we must introduce a transformation in order to apply a Hilbert space theorem. We recognize the symmetric differential and integral operators in (20) as, respectively, closed and completely continuous operators. It is possible to introduce an algebraic transformation on (20) such that the respective closed and completely continuous properties are retained, while the solutions of the transformed problem in Eqs (21)-(23) now do exist in Hilbert space. Since the transformation has destroyed the symmetry of the problem, we employ a recent extension $^{6}$ of Weyl's perturbation theorem which applies to asymmetric linear operators. This extension states that ${ }^{6}$ the addition of a completely continuous operator to a closed operator does not change the limit points of the spectrum of the closed operator. With our knowledge of the spectral properties of Eqs. (21)-(23) and in view of the symmetry of Eq. (20), it now follows that the spectrum of each $l$-component of the linearized Fokker-Planck equation contains all positive real $\lambda_{l}$; we showed earlier that the spectrum is empty for $\lambda_{l}<0$.

For $l=0$ and $l=1$ we found, respectively, twofold and three-fold degenerate zero eigenvalues, corresponding to a shift to an equilibrium different from

' I. M. Glazman, Direct Methods of Qualitative Spectral Analysis of Singular Differential Operators (Daniel Davey \& Co., New York, 1966). 
that originally postulated. For $l \geq 2$ the eigenvalues are positive and real and are $(2 l+1)$-fold degenerate, corresponding to the $2 l+1$ different spherical harmonics of order $l$.

\section{THE EXPANSION THEOREM}

With the spectral properties of the kinetic equation in hand, we can now proceed to the development of an expansion theorem based on (20). Our method is essentially an extension of the theory of singular ordinary differential equations, based on a theory due to Weyl and Levinson, ${ }^{7}$ to include singular integro-differential equations with Hilbert-Schmidt kernels.

Since the eigenfunctions of the kinetic equation are bounded and continuous on every finite interval, it is natural to pursue an expansion formula for functions $u(c)$ square integrable on the interval $\Delta: 0 \leq c<\infty$. As with the earlier theory we first establish an expansion formula on a finite subinterval $\delta$ of $\Delta, \delta: a \leq c \leq b, 0<a, b<\infty$, so that the singularities of the linear operator are external to $\delta$. The expansion formula we seek is then obtained by taking $\delta \rightarrow \Delta$ in a suitable manner. In the following we mean by $L_{\Delta}$ the integro-differential operator in (20) and by $L_{\delta}$ the operator obtained when the lower and upper limits of the integral in (20) are replaced by $a$ and $b$, respectively. In the following, the index $l$ is retained only where it is necessary to avoid confusion.

We have already seen that $P, P^{\prime}$, and $Q_{l}$ are continuous on $\delta$, and that $K_{l}\left(c, c^{\prime}\right)$ is bounded and integrable on the square $a \leq c \leq b, a \leq c^{\prime} \leq b$. Tamarkin $^{8}$ has shown that, subject to these conditions, the solutions of $L_{\delta} \phi=-\lambda \phi$ which satisfy homogeneous boundary conditions at $c=a$ and $c=b$ form a complete orthogonal and normalizable set of eigenfunctions $\left(h_{\delta n}\right)$ on $\delta$, with an associated denumerable sequence of real eigenvalues $\left(\lambda_{\delta n}\right)$. Assuming the $h_{\delta n}$ to be normalized, the expansion formula on $\delta$ is thus

$$
u(c)=\sum_{n=0}^{\infty} h_{\delta n} \int_{a}^{b} d c u(c) h_{\delta n}^{*}(c),
$$

where $u(c)$ is any function square integrable on $\delta$.

We now use the Weyl-Levinson theory to take $\delta \rightarrow \Delta$. Since the subsequent development of the expansion theorem is in every respect a duplication of the earlier theory, we display only the salient features.

${ }^{7}$ E. A. Coddington and N. Levinson, Theory of Ordinary Differential Equations (McGraw-Hill Book Company, Inc., New York, 1955), Chap. 9.

8 J. D. Tamarkin, Trans. Am. Math. Soc. 29, 755 (1927).
Given $\lambda$, the most general solution of $L_{\delta} \phi=-\lambda \phi$ is a linear combination of the two linearly independent solutions, say $\phi_{1}, \phi_{2}$. Thus, we can write

$$
h_{\delta n}\left(c, \lambda_{\delta n}\right)=r_{\delta n 1} \phi_{1}\left(c, \lambda_{\delta n}\right)+r_{\delta n 2} \phi_{2}\left(c, \lambda_{\delta n}\right),
$$

where $r_{\delta n 1}$ and $r_{\delta n 2}$ are complex constants. With (27), (26) becomes

$$
u(c)=\sum_{n=0}^{\infty} \sum_{j, k=1}^{2} r_{\delta n j} r_{\delta n k}^{*} \phi_{j} \int_{a}^{b} d c u(c) \phi_{k}^{*} .
$$

Following Levinson ${ }^{7}$ we define an Hermitian, positive semidefinite matrix $\rho_{\delta}$, called the spectral matrix, with elements $\rho_{\delta j k}$ which consist of step functions with jumps at the eigenvalues $\lambda_{\delta n}$ given by

$$
\rho_{\delta j k}\left(\lambda_{\delta n}+0\right)-\rho_{\delta j k}\left(\lambda_{\delta n}-0\right)=r_{\delta n j} r_{\delta n k}^{*} .
$$

Let $\rho_{\delta}(\lambda+0)=\rho_{\delta}(\lambda)$, and let $\rho_{\delta}(0)$ be the zero matrix. We use the spectral matrix to replace the infinite series in (28) by a Lebesgue-Stieltjes integral

where

$$
u(c)=\int_{-\infty}^{\infty} \sum_{j, k=1}^{2} \phi_{j}(c, \lambda) \tilde{u}_{k}(\lambda) d \rho_{\delta j k}(\lambda)
$$

$$
\tilde{u}_{k}=\int_{a}^{b} d c u(c) \phi_{k}^{*}(c, \lambda)
$$

As $\delta \rightarrow \Delta$ (that is, $a \rightarrow 0, b \rightarrow \infty$ ), $\rho_{\delta}$ approaches a limit matrix $\rho_{\Delta}$. To find $\rho_{\Delta}$, let $\lambda=\mu+i \omega, \omega>0$, and let $\chi_{a}=\phi_{1}+m_{a}(\lambda) \phi_{2}$ be a solution of $L_{\delta} \phi=-\lambda \phi$ satisfying the homogeneous boundary condition

$$
\cos \alpha \chi(a)+\sin \alpha P(a) \chi^{\prime}(a)=0,
$$

and similarly let $\chi_{b}=\phi_{1}+m_{b}(\lambda) \phi_{2}$ be a solution of the same equation satisfying

$$
\cos \beta \chi(b)+\sin \beta P(b) \chi^{\prime}(b)=0 .
$$

Clearly $m_{a}=r_{\delta n 2}(a) / r_{\delta n 1}(a)$ and similarly for $m_{b}$. As $a \rightarrow 0$ and $b \rightarrow \infty, m_{a}$ and $m_{b}$ approach limiting values in the complex $m$ plane denoted, respectively, by $m_{0}(\lambda)$ and $m_{\infty}(\lambda)$. These limiting values are clearly determined by the behavior of $\phi_{1}$ and $\phi_{2}$ for small and large $c$, for $\lambda$ complex.

For $\phi_{1}$ and $\phi_{2}$ to be linearly independent, it is necessary and sufficient that their Wronskian equal a nonzero constant, say one;

$$
P\left(\phi_{1} \phi_{2}^{\prime}-\phi_{2} \phi_{1}^{\prime}\right)=1 \text {. }
$$

This last is satisfied if $\phi_{1}$ and $\phi_{2}$ satisfy the conditions

$$
\begin{aligned}
\phi_{1}(s, \lambda) & =\sin \sigma, & \phi_{2}(s, \lambda) & =\cos \sigma, \\
P(s) \phi_{1}^{\prime}(s, \lambda) & =-\cos \sigma, & P(s) \phi_{2}^{\prime}(s, \lambda) & =\sin \sigma,
\end{aligned}
$$

where $s$ is an interior point of $\delta$ and $0 \leq \sigma<\pi$. These conditions are also sufficient to ensure that $\phi_{1}, \phi_{2}$ are entire functions of $\lambda$ for each fixed $c$ on $\delta$ (this follows from Tamarkin ${ }^{8}$ ). 
With these properties secured, we can find $^{7}$ the limit values $m_{0}$ and $m_{\infty}$ and hence the limit matrix $\rho_{\Delta}$, whose elements are given by

$$
\rho_{\Delta j k}(\lambda)-\rho_{\Delta j k}(\eta)=\lim _{\omega \rightarrow+0} \frac{1}{\pi} \int_{\eta}^{\lambda} \operatorname{Im} M_{j k}(\mu+i \omega) d \mu,
$$

where

$$
\begin{aligned}
& M_{11}(\lambda)=\frac{1}{m_{0}-m_{\infty}}, \\
& M_{12}(\lambda)=M_{21}(\lambda)=\frac{1}{2} \frac{m_{0}+m_{\infty}}{m_{0}-m_{\infty}}, \\
& M_{22}(\lambda)=\frac{m_{0} m_{\infty}}{m_{0}-m_{\infty}} .
\end{aligned}
$$

To find the $M_{j k}$ we need asymptotic forms of $\phi_{1}$ and $\phi_{2}$ for large and small $c$. These are given by (24) and (25), as may be verified by direct substitution. Taking $\phi_{1}, \phi_{2}$ to be asymptotic, respectively, to $c^{-l}$, $c^{l+1}$ for $c$ small, we apply the homogeneous boundary condition to $\chi_{a}$ and then take $a \rightarrow 0$ to find

$$
\begin{array}{ll}
m_{0}=\infty & (l \neq 0), \\
m_{0}=-\cot \alpha & (l=0) .
\end{array}
$$

Thus for $l \neq 0$ only $M_{22}$ can have a nonzero imaginary part and, consequently, only $\phi_{2}$ contributes to the expansion formula (29). When $l=0$ both solutions are regular at $c=0$ and the limit matrix is not determined until we specify $\alpha$. The boundary condition (22) dictates the choice $\alpha=0$.

For $c$ large we take

$$
\phi_{2} \simeq A_{l} c^{\frac{3}{4}} \cos \left(\nu_{l} c^{\frac{5}{2}}-\gamma_{l}\right) \quad\left(v_{l}=4 \lambda_{l}^{\frac{1}{2}} / 5 \pi^{\frac{1}{4}}, \lambda_{l}>0\right)
$$

and find $\phi_{1}$ by integrating (30);

$$
\phi_{1} \simeq-\frac{8 c^{\frac{3}{4}} \sin \left[v_{l}\left(c^{\frac{5}{2}}-c_{0}^{\frac{5}{2}}\right)\right]}{5 \pi^{\frac{1}{2}} A_{l} \nu_{l} \cos \left(v_{l} c_{0}^{\frac{5}{2}}-\gamma_{l}\right)},
$$

where $c_{0}$ is a constant of integration. Applying the homogeneous boundary condition to $\chi_{b} \equiv \phi_{1}+$ $m_{b}(\lambda) \phi_{2}$ and then taking $b \rightarrow \infty$ with $\operatorname{Im} \lambda>0$ we find

$$
m_{\infty}=\frac{8 i \exp \left[i\left(\nu_{l} c_{0}^{\frac{5}{2}}-\gamma_{l}\right)\right]}{5 \pi^{\frac{1}{2}} A_{l}^{2} \nu_{l} \cos \left(v_{l} c_{0}^{\frac{5}{2}}-\gamma_{l}\right)} .
$$

Combining (31)-(33) we have finally

$$
d \rho\left(\lambda_{l}\right) \equiv d \rho_{\Delta 22}\left(\lambda_{l}\right)=\frac{2 d \lambda}{\pi^{\frac{5}{2}} A_{l}^{2}\left(\lambda_{l}\right) \lambda_{l}^{\frac{1}{2}}}\left(\lambda_{l}>0\right) .
$$

Since the spectrum is empty for $\lambda_{l}<0, \rho\left(\lambda_{l}\right)$ is constant on this range. The expansion formula (29) becomes

$$
u(c)=\int_{0}^{\infty} \phi_{2}\left(c, \lambda_{l}\right) \tilde{u}\left(\lambda_{l}\right) d \rho\left(\lambda_{l}\right)
$$

with

$$
\tilde{u}=\int_{0}^{\infty} u(c) \phi_{2}^{*}\left(c, \lambda_{l}\right) d c
$$

The expansion converges in the mean for all functions $u(c)$ square integrable on $(0, \infty)$. If the spectral function $\rho$ is not continuous at $\lambda_{l}=0$, this point will contribute to the integral in (35).

We return to the description of perturbations from equilibrium. If $\exp \left(-c^{2}\right) f(c, 0)$ is square integrable in velocity space, then from (19) and (35) we have

$$
e^{-c^{2}} f(\mathbf{c}, \tau)=\sum_{l, m} \int_{0}^{\infty} d \rho\left(\lambda_{l}\right) \Psi_{l m} \tilde{F}_{l m} e^{-\lambda_{l} \tau}
$$

with

$$
\tilde{F}_{l m}\left(\lambda_{l}\right)=\frac{1}{2}(2 l+1) \int d^{3} c \Psi_{l m}^{*} f(\mathbf{c}, 0)
$$

and

$$
\Psi_{l m}\left(\mathbf{c}, \lambda_{l}\right)=c^{-1} e^{-c^{2} / 2} \psi_{l}\left(c, \lambda_{l}\right) Y_{l}^{m}(\theta, \phi) .
$$

The functions $\psi_{l}$ correspond to $\phi_{2}$ in (35) and are the solutions of (20) satisfying (22).

We have defined the density, mean velocity, and kinetic temperature of the ion gas as being proportional respectively to the first three moments of the equilibrium distribution $F_{0}$. If $\rho$ is continuous at $\lambda_{l}=0$ this point does not contribute to the expansion formula (36) and the eigenfunctions (9) for $\lambda_{l}=0$ are not contained in the expansion. By virtue of the conservation laws, the functions (9) are then orthogonal to (36). Thus (36) is complete only if $\rho$ has a jump at $\lambda_{l}=0$ for $l=0,1$. It follows that the exclusion of (9) from (36) yields an expansion which is complete with respect to all square integrable perturbations conserving $N_{0},\langle\mathbf{v}\rangle$, and $k T$.

\section{THE ELECTRON KINETIC EQUATION}

We have developed an expansion theorem based on the uncoupled kinetic equation (3) for the ions. The extension to the electron kinetic equation is straightforward and requires only a little algebra.

As indicated earlier, we decouple the electron kinetic equation from the ion equation by dropping the term $\left(m_{e} / m_{i}\right) \partial f_{i}^{\prime} / \partial \mathbf{v}^{\prime}$ in (2). This amounts to neglecting the effect of the ion perturbation on the electron perturbation, but retains the effects of encounters with ions in the thermal distribution.

The uncoupled equation conserves electron number density provided (4) holds, but does not conserve momentum or kinetic energy in the electron gas. This is as it should be, since a substantial portion of the electron momentum, and a small amount of the energy, is lost to the ions. 
Applying the methods of Sec. I we find as before $\operatorname{Re} \lambda \geq 0, \operatorname{Im} \lambda=0$, and for $\lambda=0$ we find $g_{0}=$ const, corresponding to (9). The remainder of the development proceeds as before. A spherical harmonic expansion yields a set of singular integro-differential equations, and the transformation (19) brings these into self-adjoint form. As before, the expansion formula has the form of a Riemann-Stieltjes, or generalized Fourier, integral.

If, for example, the ions are protons, then we can take $\Gamma_{e e}=\Gamma_{e i}$. If we use $\alpha_{e}$ in place of $\alpha_{i}$ in the definition of $\tau$ and $c$, then the electron equations may be obtained from the ion equations by replacing $T(c)$ in (15) by $T(c)+\alpha^{\frac{1}{2}} T\left(\alpha^{-\frac{1}{2}} c\right)$, where $\alpha \equiv \alpha_{e} / \alpha_{i}=m_{e} / m_{i}$.

\section{DISCUSSION}

We have used boundary conditions obtained by requiring the solutions of the kinetic equation to be consistent with the conservation laws. The Hilbert space then 'emerged as a natural function space for the framework of the mathematical development. The question persists (see, e.g., the discussion in Uhlenbeck and Ford ${ }^{9}$ ) as to whether square integrability should be a requirement on the distribution functions from the beginning. In the light of the present work, this condition does not appear to be necessary, and for our purposes, it would not have been sufficient. To see this we note the condition $\int d^{3} v\left|F_{0} f\right|^{2}<\infty$ leads to

$$
\lim _{v \rightarrow 0} v^{\frac{3}{2}} f=0
$$

which is weaker than the corresponding condition (4). Since both solutions of (20) satisfy (37) for $l=0$, it would be possible to have an expansion theorem for solutions of the kinetic equation which are square integrable but do not satisfy the conservation laws.

\section{ACKNOWLEDGMENTS}

The author is indebted to Professor R. K. Osborn, Professor C. L. Dolph, and Professor F. C. Shure for many helpful discussions during the course of this work, which represents part of a Ph.D. dissertation submitted to the university of Michigan (1966).

This research was supported in part by the United States Atomic Energy Commission and by the Advanced Research Projects Agency, Project Defender, ARPA Order No. 675, was monitored by the United States Army Research Office (Durham) under Contract DA-31-124-ARO-D-403.

\footnotetext{
- G. E. Uhlenbeck and G. W. Ford, Lectures in Statistical Mechanics (American Mathematical Society, Providence, Rhode Island, 1963), p. 88.
}

\section{APPENDIX A. INTEGRALS}

Let $\Theta$ be the angle between $c$ and $\mathbf{c}^{\prime}$. From the generating function relation for Legendre polynomials, we have

$$
\begin{aligned}
\left|\mathbf{c}-\mathbf{c}^{\prime}\right|^{-1} & =\left(c^{2}+c^{\prime 2}-2 c c^{\prime} \cos \Theta\right)^{-\frac{1}{2}} \\
& =c^{-1} \sum_{l=0}^{\infty}\left(\frac{c^{\prime}}{c}\right)^{l} P_{l}(\cos \Theta) \quad\left(c^{\prime} \leq c\right),
\end{aligned}
$$

and similarly for $c \leq c^{\prime}$. Writing

$$
g_{\lambda}(\mathbf{c})=\sum g_{l m} Y_{l}^{m}
$$

and employing the addition theorem for Legendre polynomials, we obtain Eq. (13).

To find Eq. (14) we use the relation

$$
\begin{aligned}
\left(1+x^{2}-2 x y\right)^{\frac{1}{2}}= & \int d x x\left(1+x^{2}-2 x y\right)^{-\frac{1}{2}} \\
& -y \int d x\left(1+x^{2}-2 x y\right)^{-\frac{1}{2}}
\end{aligned}
$$

Combining (A1) and (A2) we have

$$
\begin{aligned}
&\left|\mathbf{c}-\mathbf{c}^{\prime}\right|=\left(c^{2}+c^{\prime 2}-2 c c^{\prime} \cos \Theta\right)^{\frac{1}{2}} \\
&=c^{-1} \sum_{l=0}^{\infty}\left(\frac{\left(c^{\prime} / c\right)^{l+2}}{l+2}-\frac{\left(c^{\prime} / c\right)^{l+1}}{l+1} \cos \Theta\right) P_{l}(\cos \Theta) \\
&\left(c^{\prime} \leq c\right),
\end{aligned}
$$

and similarly for $c \leq c^{\prime}$. From the pure recurrence relation for Legendre polynomials, we have

$$
\begin{aligned}
\cos \Theta P_{l}(\cos \Theta)=\frac{l}{2 l+1} & P_{l-1}(\cos \Theta) \\
& +\frac{l+1}{2 l+1} P_{l+1}(\cos \Theta)
\end{aligned}
$$

Combining (A3) and (A4) and then using the addition theorem as before we find $\mathrm{Eq}$. (14).

Finally, Eq. (15) is obtained from Eq. (14) with $g_{l m}=\delta_{l 0} \delta_{m 0}$ with $\delta_{j k}$ the Kronecker delta.

\section{APPENDIX B. THE SELF-ADJOINT PROPERTY}

Let $L_{\delta}$ be either (i) the differential operator in Eq. (20) or (ii) the integro-differential operator in Eq. (20) defined as in Sec. IV on the closed interval $\delta$ : $a \leq c \leq b, 0<a, b<\infty$. Let $L_{\Delta}$ be similarly defined on $(0, \infty)$. Then in either case (i) or case (ii) there exists a complete orthonormal set of functions $\left\{h_{\delta n}\right\}$ on $\delta$, generated by $L_{\delta} \phi=-\lambda \phi$ with homogeneous boundary conditions at $a$ and $b .^{7.8}$

Let $f$ and $g$ be any nonzero functions square integrable on $(0, \infty)$, and consider the inhomogeneous 
problems

$$
L_{\delta} u+\Lambda u=f, \quad L_{\delta} v+\Lambda^{*} v=g
$$

with the same homogeneous boundary conditions at $a$ and $b$ as in the above homogeneous problem. Let $\operatorname{Im} \Lambda \neq 0$ so that $\Lambda$ will not belong to the spectrum of the set $\left\{h_{\delta n}\right\}$. Then the problems (B1) have nontrivial solutions $^{8}$

$$
\begin{aligned}
& u(c)=\int_{-\infty}^{\infty} \sum_{j, k=1}^{2} \frac{\phi_{j}(c, \lambda) \tilde{u}_{k}(\lambda) d \rho_{\delta j k}(\lambda)}{\Lambda-\lambda}, \\
& v(c)=\int_{-\infty}^{\infty} \sum_{j, k=1}^{2} \frac{\phi_{j}(c, \lambda) \tilde{v}_{k}(\lambda) d \rho_{\delta j k}(\lambda)}{\Lambda^{*}-\lambda},
\end{aligned}
$$

with

$\tilde{u}_{k}=\int_{a}^{b} f(c) \phi_{k}^{*}(c, \lambda) d c, \quad \tilde{v}_{k}=\int_{a}^{b} g(c) \phi_{k}^{*}(c, \lambda) d c$.

Now, let

$$
\begin{aligned}
& u_{\mu}=\int_{-\mu}^{\mu} \sum_{j, k=1}^{2} \frac{\phi_{j} \tilde{u}_{k} d \rho_{\delta j k}}{\Lambda-\lambda}, \\
& v_{\mu}=\int_{-\mu}^{\mu} \sum_{j, k=1}^{2} \frac{\phi_{j} \tilde{v}_{k} d \rho_{\delta j k}}{\Lambda^{*}-\lambda},
\end{aligned}
$$

with $\tilde{u}_{k}$ and $\tilde{v}_{k}$ as in (B2). Multiplying $u_{\mu}$ by $g^{*}$ and $v_{\mu}^{*} \quad$ which is the desired result. by $f$ and then integrating over $\delta$ we have

$$
\begin{aligned}
\int_{a}^{b} g^{*}(c) & u_{\mu}(c) d c \\
& =\int_{-\mu}^{\mu} \sum_{j, k=1}^{2} \frac{\tilde{u}_{k}(\lambda) d \rho_{\delta j_{k}}(\lambda)}{\Lambda-\lambda} \int_{a}^{b} g^{*}(c) \phi_{j}(c, \lambda) d c \\
& =\int_{-\mu}^{\mu} \sum_{j, k=1}^{2} \frac{\check{u}_{k}(\lambda) \tilde{v}_{j}^{*}(\lambda) d \rho_{\delta j k}(\lambda)}{\Lambda-\lambda},
\end{aligned}
$$

and similarly

$$
\int_{a}^{b} f(c) v_{\mu}^{*}(c) d c=\int_{-\mu}^{\mu} \sum_{j, k=1}^{2} \frac{\tilde{v}_{k}^{*}(\lambda) \tilde{u}_{j}(\lambda) d \rho_{\delta j k}(\lambda)}{\Lambda-\lambda} .
$$

Since (24) and (25) are asymptotic solutions for both the differential and the integro-differential equations, the limit matrix $\rho_{\Delta}$ is the same in either case. Taking $\delta \rightarrow \Delta$ in (B3) and (B4) we have $\rho_{\delta j k} \rightarrow \rho_{\Delta 22}$ and thus

$$
\int_{0}^{\infty} g^{*}(c) u_{\mu}(c) d c=\int_{-\mu}^{\mu} \frac{\tilde{u}_{2} \hat{v}_{2}^{*} d \rho_{\Delta 22}}{\Lambda-\lambda}=\int_{0}^{\infty} f(c) v_{\mu}^{*}(c) d c
$$

After taking $\mu \rightarrow \infty$ and employing (B1), (B5) becomes

$$
\int_{0}^{\infty}\left(L_{\Delta} v\right)^{*} u d c=\int_{0}^{\infty}\left(L_{\Delta} u\right) v^{*} d c
$$

\title{
Real Spinor Fields
}

\author{
David Hestenes* \\ Palmer Laboratory, Princeton University, Princeton, New Jersey
}

(Received 22 March 1966)

\begin{abstract}
The Dirac equation is expressed entirely in terms of geometrical quantities by providing a geometrical interpretation for the $(-1)^{\frac{1}{2}}$ which appears explicitly in the Dirac equation. In the modification of the Dirac electron theory which ensues, the $(-1) \frac{1}{2}$ appears as the generator of rotations in the spacelike plane orthogonal to the plane containing the electron current and spin vectors. This amounts to a further "relativistic" constraint on the spinor theory and so may be expected to have physical consequences. It does not, however, conflict with well-substantiated features of the Dirac theory.
\end{abstract}

\section{INTRODUCTION}

$\mathrm{I}^{\mathrm{N}}$ N 1928, Dirac proposed a relativistically invariant first-order wave equation for the electron. ${ }^{1}$ Dirac's theory has led to a complex of physical explanations and predictions at once so surprising and convincing that it has gained general acceptance among physicists today. The previously perplexing phenomena of

\footnotetext{
* Present address: Physics Department, Arizona State University, Tempe, Arizona.

1 P. A. M. Dirac, Proc. Roy. Soc. (London) 117, 610 (1928).
}

electron spin was not only accounted for, but fine details of the hydrogen spectra and an accurate value of the electron magnetic moment were calculated without arbitrary assumptions. Moreover, after some theoretical trauma, it was realized that Dirac's equation entails the existence of a positively charged electron-at just about the same time that such a particle was discovered experimentally. We do well to understand precisely what features of the Dirac equation entail these remarkable results. 